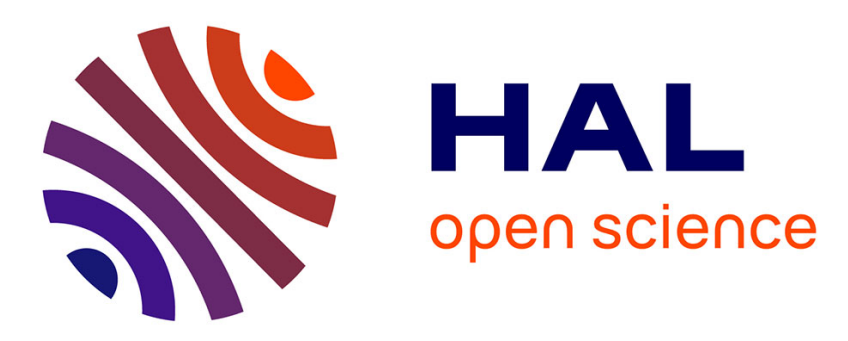

\title{
Energy/Reliability Trade-off of LoRa Communications over Fading Channels
}

Xuan-Chien Le, Baptiste Vrigneau, Matthieu Gautier, Malo Mabon, Olivier Berder

\section{- To cite this version:}

Xuan-Chien Le, Baptiste Vrigneau, Matthieu Gautier, Malo Mabon, Olivier Berder. Energy/Reliability Trade-off of LoRa Communications over Fading Channels. International Conference on Telecommunication, Jun 2018, Saint-Malo, France. hal-01816574

\section{HAL Id: hal-01816574 \\ https://hal.science/hal-01816574}

Submitted on 15 Jun 2018

HAL is a multi-disciplinary open access archive for the deposit and dissemination of scientific research documents, whether they are published or not. The documents may come from teaching and research institutions in France or abroad, or from public or private research centers.
L'archive ouverte pluridisciplinaire HAL, est destinée au dépôt et à la diffusion de documents scientifiques de niveau recherche, publiés ou non, émanant des établissements d'enseignement et de recherche français ou étrangers, des laboratoires publics ou privés. 


\title{
Energy/Reliability Trade-off of LoRa Communications over Fading Channels
}

\author{
Xuan-Chien Le, Baptiste Vrigneau, Matthieu Gautier, Malo Mabon, Olivier Berder \\ Univ Rennes, CNRS, IRISA \\ 6 rue de Kerampont, 22300 Lannion, France \\ \{xuan-chien.le, baptiste.vrigneau, matthieu.gautier, malo.mabon, olivier.berder\}@ irisa.fr
}

\begin{abstract}
LoRa is a technology for long range wireless communications that allows the development of new applications in domains such as smart agriculture, smart city or smart industry. Many in-field deployments and measurement campaigns have been performed in recent years, showing the sensibility of such communication to fading channels. In this paper, the LoRa transmission reliability is evaluated in simulations for different fading channels. Moreover, as the energy consumption also depends on the configurations, a trade-off between energy consumption and reliability needs to be considered when selecting a LoRa configuration. To this aim, experimental energy measurements are performed on a Semtech device, showing that energy difference between various configurations can reach up to two orders of magnitude. Results highlight that LoRa configuration impacts the energy/reliability trade-off and the best one strongly depends of the type of channel.
\end{abstract}

\section{INTRODUCTION}

In recent years, Low-Power Wide-Area Networks (LPWANs) have been widely studied and deployed in many areas, particularly in smart agriculture applications, enabling large range of Internet of Things. The main advantage of LPWANs is that energy consumption is kept low for long communication ranges. This characteristic allows an end-device, powered by a battery or energy harvesting sources, to enhance its lifetime. Among various LPWAN technologies (SigFox, Adaptrum or Weightles ...), LoRa [1] physical layer has the particularity to be highly configurable regarding the spreading factor $(S F)$, bandwidth $(B W)$, coding rate $(C R)$ and transmission power $\left(P_{T x}\right)$, which are critical parameters permitting a trade-off between energy consumption, data rate and transmission range.

Many studies address this flexibility in the LoRa configuration choice and the resulting performance trade-offs. An experimental analysis is performed in [2] to evaluate the coverage capacity of a base station in LoRa-based LPWAN using the LoRa default configuration. An area of $1380 \mathrm{~km}^{2}$ can potentially be covered by one base station located $470 \mathrm{~m}$ above the sea level. Another study [3] experimentally evaluates the LoRa network coverage in a suburban area. It is shown that the range can be increased from $650 \mathrm{~m}$ to $3.4 \mathrm{~km}$ by changing the configuration. In [4], the trade-off between the energy consumption and the sensibility in LoRa communications is studied. Experimental results show that if the consumed energy is reduced when using configurations with high data rate, the communication range also decreases. The sensibility to device mobility is studied in [5]. Measurement campaigns highlight that the LoRa modulation is reliable when the device velocity is maintained lower than a threshold depending on the configuration, e.g. $38 \mathrm{~km} / \mathrm{h}$ with $S F=12$ and $B W=125 \mathrm{kHz}$. Moreover, lower $S F$ s allow higher velocities. Meanwhile, in [6], the limitations of LoRaWAN are studied in different use cases, which require different types of data transmission patterns, latency and geographical features. Apparently, the constraints on delay and data rate prevent this technology from applying in some applications such as smart transportation and logistics or video surveillance, while small SFs are selected to reduce the time on air when deploying LoRaWAN for agriculture real time monitoring.

Therefore, configuration selection plays an important role in any LoRa communication. This feature is already used in the LoRaWAN standard that implements an Adaptive Data Rate (ADR) scheme which adapts both end-device configuration and transmission power according to the received Signal-toNoise Ratio (SNR). However, ADR scheme does not take into account the LoRa sensitivity to fading channels and this sensitivity has just been experimentally observed in state-ofthe-art studies. In this paper, the fading channel influence is quantitatively evaluated using a Rice fading channel with a Doppler frequency. To this aim, six configurations with different data rates are considered and the reliability is imposed by a target Packet Error Rate (PER). Moreover, as the configuration also impacts the consumed energy [7], the aim of the paper is to link the reliability with the energy consumption. Thus, the energy consumption of each configuration is achieved using experimental measures on a Semtech LoRa device. Finally, the trade-off between energy consumption and reliability for LoRa communications according to Rice fading is analyzed.

In the remaining, the LoRa technology is introduced in Section II. Section III presents the channel models. Simulation results are shown in Section IV as well as the energy/reliability trade-off is discussed. Conclusions are given in Section V.

\section{LORA TECHNOLOGY}

LoRa is a long range communication technology promoted by the LoRa Alliance. The LoRa physical layer, which allows long-range, low-power and low data rate communications, is developed by Semtech [8][9]. LoRa communications can use frequency bands of $433 \mathrm{MHz}$ and $868 \mathrm{MHz}$ in Europe (respectively $915 \mathrm{MHz}$ in US and $783 \mathrm{MHz}$ in China) and its data rate can reach up to to $50 \mathrm{kbps}$. It uses the Chirp 
Spread Spectrum (CSS) modulation with a linear variation of frequency on time [10][11], which allows the reduction of both interference and Doppler effects. LoRa modulation can be configured with three parameters: $B W, S F$ and $C R$.

In LoRa, $S F$ is defined as the logarithm in base 2 of the number of chirp per symbol. LoRa operates with $S F$ from 6 to 12 . LoRa uses three bandwidths: $125 \mathrm{kHz}, 250 \mathrm{kHz}$ and $500 \mathrm{kHz}$. A LoRa symbol is therefore composed of $2^{S F}$ chirps covering the entire bandwidth, starting with a series of upward (or downward) chirps from an initial frequency, which represents for a code word. The frequency will wraps around to the minimum frequency (or maximum frequency with downchirp) when the maximum (minimum with down-chirp) of the bandwidth is reached. A linear chirp signal is usually defined by:

$$
s(t)=A \times e^{j\left(2 \pi\left(f_{0} t+\frac{\mu t^{2}}{2}\right)+\phi_{0}\right)},
$$

where $\phi_{0}$ is the initial phase, $A$ the amplitude, $\mu$ the chirp rate, $f_{0}$ the starting frequency of chirp and $\phi(t)=$ $2 \pi\left(f_{0} t+\frac{\mu t^{2}}{2}\right)+\phi_{0}$ is the instantaneous phase at time $t$. The corresponding instantaneous frequency is derived by:

$$
f(t)=\frac{d \phi(t)}{2 \pi d t}=f_{0}+\mu t
$$

Applying CSS to LoRa modulation, each frequency $f_{0}$ in the band represents a code word. The frequency is swapped at the maximum value of bandwidth, the instantaneous frequency is therefore represented as a modulo operator of $B W$, given by:

$$
f(t)=f_{\min }+\left[\left(\Delta f_{0}+\mu t\right) \bmod B W\right],
$$

where $\Delta f_{0}=f_{0}-f_{\min }$ and $f_{\min }$ is the minimum frequency of the bandwidth.

With $2^{S F}$ chirps per symbol, a code word can contain $S F$ information bits. The duration $T_{s}$ of a symbol is given by: $T_{s}=2^{S F} / B W$. For a given bandwidth, increasing the spreading factor by one unit doubles the Time-on-Air (ToA) to transmit the same amount of data, resulting in the decrease of bit rate. The bit rate is calculated by:

$$
R_{b}=S F \times \frac{B W}{2^{S F}} .
$$

Fig. 1 shows an example of LoRa transmitted symbols with different values of $S F$ from seven to twelve.

The third parameter in the configuration of LoRa modulation is the code rate. LoRa can use Forward Error Correction (FEC) code for each block of four information bits. The number of redundant bits for each block varies from one to four, corresponding to $C R \mathrm{~s}$ of $4 / 5,4 / 6,4 / 7$ and $4 / 8$. Then, the bit stream is processed by an interleaver to make FEC code more robust to burst errors. Additionally, a whitening structure can also be used to make the signal like white noise, thus avoiding frequency selective channel penalties.

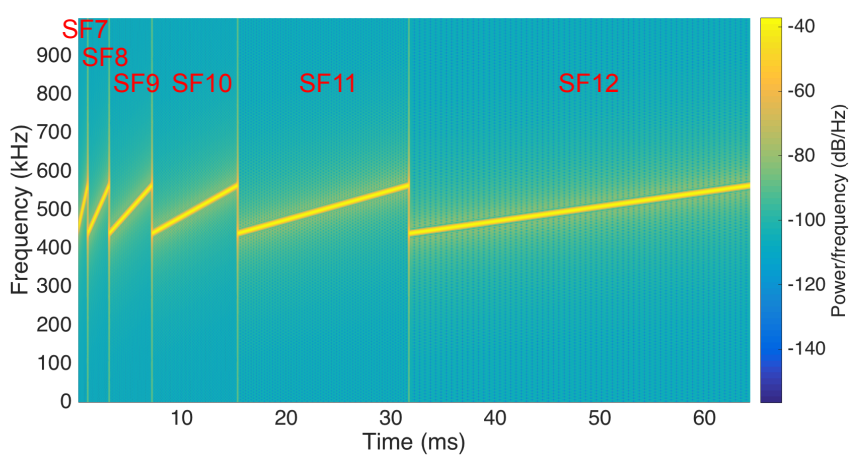

Fig. 1: LoRa transmitted symbols with different values of $S F$.

\section{CHANNEL MODELS WITH MOBILITY}

The performance of LoRa transmission is evaluated using both Rayleigh and Rice models. Moreover, Additive White Gaussian Noise (AWGN) is considered to model path-loss effects.

\section{A. Rayleigh fading channel}

In real-field deployment, the environment between the enddevice and base station has many obstacles such as buildings, trees, etc. These obstacles reflect the transmitted signal and generate other paths of transmission. The received signal at the base station is the combination of these replicas and corresponds to multi-path fading channel. In the case of Non Light-of-Sight (NLoS) paths between the end-device and base station, the channel gain $h(t)$ can be considered as a sum of in-phase and quadrature-phase components, i.e. $h(t)=h_{I}(t)+j h_{Q}(t)$. In some scattering environment, $h_{I}(t)$ and $h_{Q}(t)$ are independent Gaussian variables with zero mean and variance $\sigma_{h}^{2}$ at any time $t$. Therefore, the amplitude $|h(t)|$ follows a Rayleigh distribution. In this study, the Rayleigh fading channel is generated using the Jakes model [12] defined by:

$$
h(t)=\frac{1}{\sqrt{L}} \sum_{l=1}^{L} e^{j\left(2 \pi f_{D} \cos \left(\phi_{l}\right) t+\theta_{l}\right)},
$$

where $L$ is the number of NLoS paths, $\theta_{l}$ and $\phi_{l}$ are the initial phase and incident angle of $l$-th path, respectively. The Doppler shift $f_{D}$ is due to the movement of the end-device and can be estimated by $f_{D} \approx v_{r} c / f_{c}$, where $v_{r}$ is the relative velocity between the end-device and base station on the radius direction, $c$ is the light celerity and $f_{c}$ is the carrier frequency. The Doppler shift affects the coherence time of the channel, given by $T_{c}=\frac{1}{f_{D}}$. As a consequence, when $T_{s}>T_{c}$, or $v_{r}>$ $\frac{c B W}{f_{0} 2^{S F}}$, fast fading occurs that leads to signal distortion [5].

The channel generated by (5) is normalized by $1 / \sqrt{L}$ to normalize the average gain. The initial phase $\theta_{l}$ is assumed statistically independent and uniformly distributed in $[0,2 \pi)$. 
In Jakes model, the incident signal is assumed to come from all directions. Therefore, if assigning $\psi_{0}$ as a random value uniformly chosen in $[0,2 \pi)$, then $\phi_{l}$ is given by

$$
\phi_{l}=\psi_{0}+2 \pi \frac{l-1}{L} \quad \text { with } l=1,2, \ldots, L .
$$

\section{B. Rice fading channel}

In the case of LoS environment, $h_{I}(t)$ and $h_{Q}(t)$ are independent Gaussian variables but with non-zero means, denoted $\bar{h}_{I}(t)$ and $\bar{h}_{Q}(t)$, respectively. Besides, $h_{I}(t)$ and $h_{Q}(t)$ are supposed to have the same variance $\sigma_{h}^{2}$ at any time $t$. Then, the amplitude of $h(t)$ follows a Rice distribution. A method to determine the value of $\bar{h}_{I}(t)$ and $\bar{h}_{Q}(t)$ is introduced in [13], in which $\bar{h}_{I}(t)$ and $\bar{h}_{Q}(t)$ are modeled as deterministic timevarying parameters:

$$
\begin{aligned}
\bar{h}_{I}(t) & =\chi \cos \left(2 \pi f_{D} \cos \left(\phi_{0}\right) t+\theta_{0}\right) \\
\bar{h}_{Q}(t) & =\chi \sin \left(2 \pi f_{D} \cos \left(\phi_{0}\right) t+\theta_{0}\right),
\end{aligned}
$$

where $\chi^{2}=\bar{h}_{I}(t)^{2}+\bar{h}_{Q}(t)^{2}$ is the non-centrality parameter of Rice distribution, $f_{D} \cos \left(\phi_{0}\right)$ and $\theta_{0}$ are the Doppler frequency and the phase of the LoS path, respectively.

The Rice fading channel can be set with the Rice factor $K$, defined as the ratio between the power of the direct path $\chi^{2}$ and the power of NLoS paths $2 \sigma_{h}^{2}$, i.e. $K=\frac{\chi^{2}}{2 \sigma_{h}^{2}}$. When $K=0$, the channel becomes a Rayleigh channel, while when $K=\infty$, the channel does not have fading phenomenon anymore. Without loss of generality, the average power of the channel is normalized to 1 and the power of LoS and NLoS paths can be defined with the Rice factor as:

$$
\begin{aligned}
\chi^{2} & =\frac{K}{K+1} \\
2 \sigma_{h}^{2} & =\frac{1}{K+1} .
\end{aligned}
$$

Therefore, a Rice fading channel is composed of two components: the first one is generated by the NLoS paths and follows Rayleigh distribution with power $2 \sigma_{h}^{2}=1 /(K+1)$; the second one is the LoS path with deterministic time-varying parameters $\bar{h}_{I}(t)$ and $\bar{h}_{Q}(t)$ calculated by (7) and (8), with power $\chi^{2}=K /(K+1)$. The first scattered component $h_{\mathrm{NLoS}}(t)$ is generated by (5) and normalized in order to get the power of $1 /(K+1)$, and the second part $h_{\mathrm{LoS}}(t)$ corresponds to the LoS component determined by $h_{\mathrm{LoS}}(t)=\bar{h}_{I}(t)+j \bar{h}_{Q}(t)$. As a conclusion, the Rice channel gain can be obtained by:

$$
h(t)=\frac{1}{\sqrt{K+1}}\left[h_{\mathrm{NLoS}}(t)+\sqrt{K} e^{j\left[2 \pi f_{D} \cos \left(\phi_{0}\right)+\theta_{0}\right.}\right],
$$

with $\phi_{0}$ and $\theta_{0}$ uniformly distributed in $[0,2 \pi)$.

\section{LoRA TRAnSMission Simulation}

\section{A. LoRa Setup}

To evaluate the transmission performance, six configurations of LoRa are used for both experiments and simulations. The configurations are detailed in Table I, the first one has the lowest data rate, while the last one has the highest. Configuration 3 is the LoRa default setup. For the simulations,
TABLE I: LoRa configuration parameters and energy measurements for different transmission power levels.

\begin{tabular}{c||c|c|c|c|c|c} 
Configuration & 1 & 2 & 3 & 4 & 5 & 6 \\
\hline \hline$C R$ & $4 / 8$ & $4 / 5$ & $4 / 5$ & $4 / 5$ & $4 / 8$ & $4 / 5$ \\
\hline$S F$ & 12 & 8 & 7 & 7 & 7 & 6 \\
\hline$B W(\mathrm{kHz})$ & 125 & 125 & 125 & 500 & 500 & 500 \\
\hline$R_{b}(\mathrm{kbps})$ & 0.37 & 3.91 & 6.84 & 27.34 & 27.34 & 46.88 \\
\hline $\mathrm{ToA}(\mathrm{ms})$ & 1712.13 & 102.91 & 56.58 & 14.14 & 19.52 & 7.71 \\
\hline \hline $\begin{array}{c}\mathrm{E}(\mathrm{mJ}) \\
\left(P_{T x}=2 \mathrm{dBm}\right)\end{array}$ & 240 & 14.43 & 7.9 & 2.01 & 2.77 & 1.1 \\
\hline $\begin{array}{c}\mathrm{E}(\mathrm{mJ}) \\
\left(P_{T x}=10 \mathrm{dBm}\right)\end{array}$ & 379.12 & 22.74 & 12.41 & 3.15 & 4.32 & 1.72 \\
\hline $\begin{array}{c}\mathrm{E}(\mathrm{mJ}) \\
\left(P_{T x}=14 \mathrm{dBm}\right)\end{array}$ & 507.37 & 31.96 & 17.45 & 4.55 & 6.13 & 2.48
\end{tabular}

the performance of LoRa is measured in term of PER, 2000 packets with 25 bytes of payload per packet are transmitted for each configuration.

\section{B. Energy measurements}

First, energy measurements have been performed to estimate the energy per transmitted packet, a payload of 25 bytes per packet is considered. This energy is measured for each configuration and for three levels of $P_{T x}: 2 \mathrm{dBm}, 10 \mathrm{dBm}$ and $14 \mathrm{dBm}$. The experimental setup consists in a LoRa enddevice prototype and a high sensitive current analyzer. The prototype is composed of a Texas Instrument MSP430FR6989 micro-controller and a Semtech SX1276 transceiver. The measurements were obtained using an Keysight CX3324A current waveform analyzer (1 GSample/s, $100 \mathrm{pA}$ accuracy). Fig. 2 shows an example of the current waveform when using the configuration 5 . The different steps can be observed from the sleep mode to the transmission mode. Latency due to turning on the micro-controller and configuring the transceiver can be

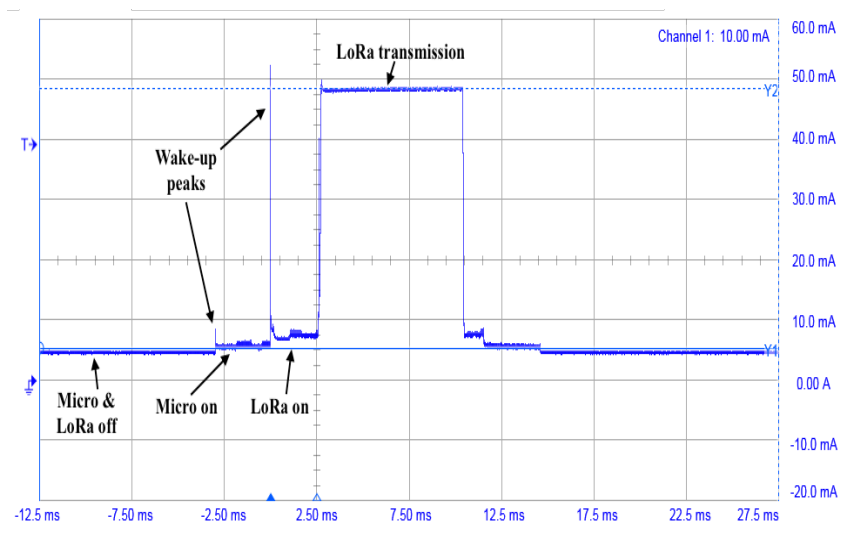

Fig. 2: Microbenchmark showing the current waveform of transmitting a packet with configuration 5 . 


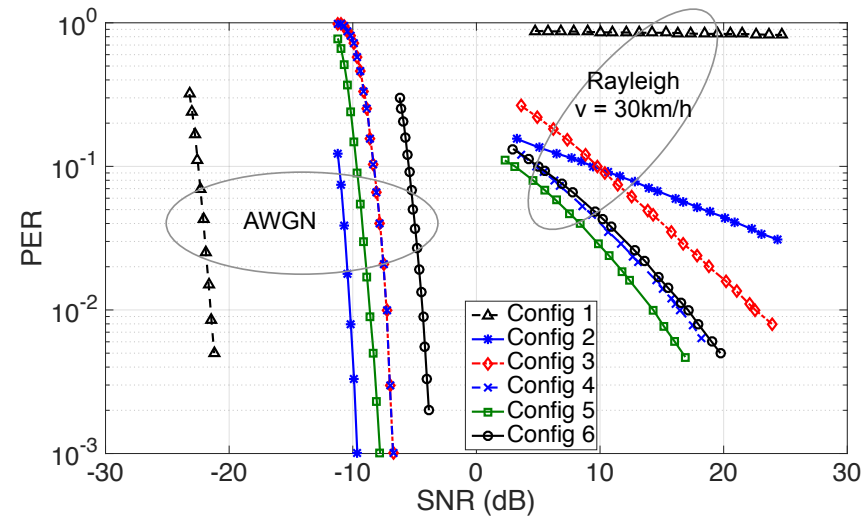

Fig. 3: LoRa performance in AWGN channel and Rayleigh channel (with an end-device velocity of $30 \mathrm{~km} / \mathrm{h}$ ).

observed as well as high current peaks that must be taken into account when powering the platform.

Energy measurement results are shown in Table I. The energy required to send a packet using the highest data rate is 200 times lower than the one required to send a packet using the lowest data rate. The energy per packet is obviously increasing with the transmission power, about twice more between $2 \mathrm{dBm}$ and $14 \mathrm{dBm}$.

\section{Reliability evaluation}

To evaluate the LoRa reliability over different channels, performance in terms of PER is obtained using simulations for all the configurations. The results are shown is Fig. 3 for both AWGN channel and Rayleigh channel with enddevice velocity of $30 \mathrm{~km} / \mathrm{h}$, PER are given according to the Signal-to-Noise Ratio (SNR) at the receiver. In a non-fading AWGN channel, LoRa transmission using the first configuration $(S F 12,125 \mathrm{kHz}$ of $B W$ ) shows the best performance, and can reach a PER of $10^{-1}$ at SNR $=-22.5 \mathrm{~dB}$ and $10^{-2}$ at $\mathrm{SNR}=-21.5 \mathrm{~dB}$. Meanwhile, configuration $6(S F 6,500 \mathrm{kHz}$ of $B W$ ) shows the worst performance with a PER of $10^{-1}$ at $\mathrm{SNR}=-5.47 \mathrm{~dB}$ and $10^{-2}$ at $\mathrm{SNR}=-4.36 \mathrm{~dB}$. In AWGN channel, performance is globally better when increasing the spreading factor. On the other hand, the bandwidth does not affect the performance as configuration $3(S F 7,125 \mathrm{kHz}$ of $B W, C R 4 / 5)$ and configuration $4(S F 7,125 \mathrm{kHz}$ of $B W$, $C R$ 4/5) achieve the same performance. Finally, using a FEC code improves the performance and a $1.4 \mathrm{~dB}$ gain at PER = $10^{-1}$ can be achieved between configuration $4(S F 7,125 \mathrm{kHz}$ of $B W, C R 4 / 5)$ and configuration $5(S F 7,125 \mathrm{kHz}$ of $B W$, $C R$ 4/8).

In contrast, when Rayleigh fading channel is considered, the first configuration shows the worst performance with a PER always higher than $80 \%$. This result can be explained by the symbol time of this configuration close to the coherence time of the channel at $30 \mathrm{~km} / \mathrm{h}$, i.e. $T_{s}=32.8 \mathrm{~ms}$ and $T_{c} \approx 41.5 \mathrm{~ms}$, respectively. The long symbol duration of configuration 1 makes it highly sensitive to fading channel.

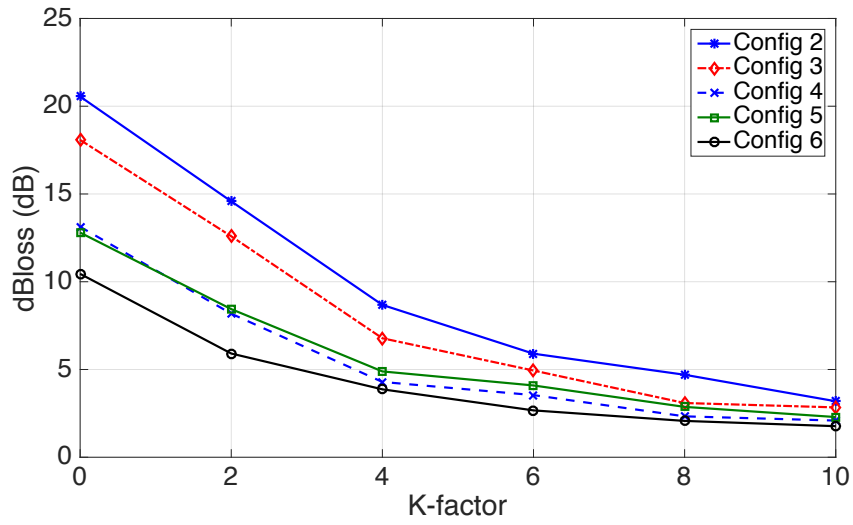

Fig. 4: Loss of received signal power to maintain $P E R=10^{-1}$ as in AWGN channel when the end-device velocity is $30 \mathrm{~km} / \mathrm{h}$ ).

Similarly, configuration 2 with a $S F$ of 8 also shows a bad performance, with a $20.55 \mathrm{~dB}$ loss compared to AWGN channel to reach a PER of $10^{-1}$ and the inability to obtain PER of $10^{-2}$. While configurations 3 and 4 reach the same performance in an AWGN channel, configuration 4 outperforms configuration 3 in a Rayleigh fading channel. Using a larger bandwidth $(500 \mathrm{kHz}$ compared to $125 \mathrm{kHz}$ for configuration 3) reduces the symbol duration and makes the transmission less disturbed by the fading.

To evaluate the performance changes of each configuration due to the channel variation, we defined the dBloss as the loss of received signal power to obtain the same PER as for AWGN channel. The dBloss are given in Fig. 4 for PER $=10^{-1}$ for different values of the $K$-factor in Rice channel model. When $K \rightarrow+\infty$, the channel can be considered as AWGN and the dBloss decreases to $0 \mathrm{~dB}$ for all configurations. Because PER $=10^{-1}$ can not be obtained with configuration 1, Fig. 4 only shows the results of five remaining configurations. The simulation results show that configuration 2 always obtains the highest dBloss, while configuration 6 gives the lowest. Meanwhile, configurations 4 and 5 get the same loss, meaning that they have the same sensitivity to fading channel. In a general way, configurations with higher spreading factor, i.e longer symbol duration, are more sensitive to the channel variation.

\section{Energy-reliability trade-off}

As illustrated in Table I and Fig. 3, a configuration with higher data rate consumes less energy but gives a worse performance in AWGN channel. Therefore, the selection of a configuration has to make balance between the energy consumption and the reliability depending on the fading channel. Fig. 5 illustrates the energy consumption for $14 \mathrm{dBm}$ transmission power to send 25 bytes of payload and the minimum SNR at the receiver to obtain a target PER of $10^{-1}$ over different channels. Configurations 1 and 2 seem to be less efficient. While the PER of $10^{-1}$ can not be obtained with configuration 1 , configuration 2 requires too 


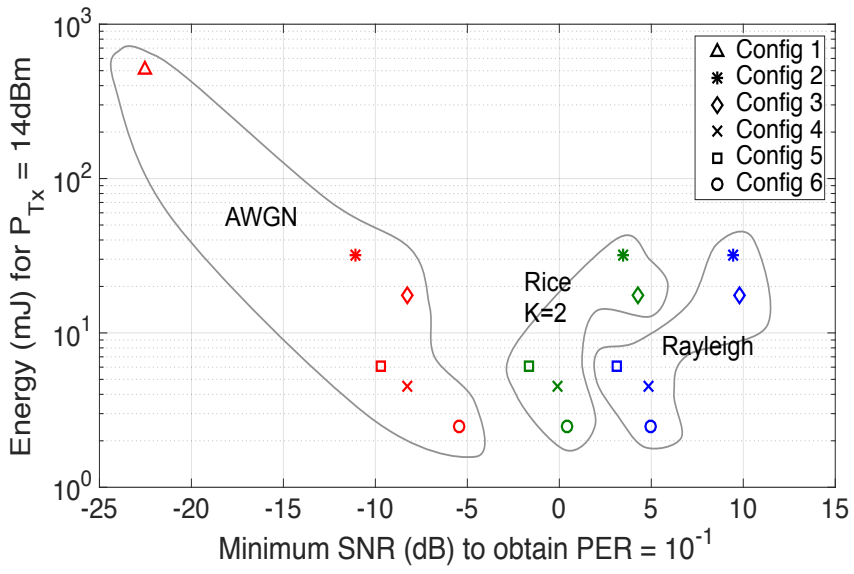

Fig. 5: Energy consumption $\left(P_{T x}=14 \mathrm{dBm}\right)$ versus minimum SNR at the receiver to obtain PER of $10^{-1}$.

much energy and high SNR at the receiver to obtain the target PER in Rayleigh fading channel. In contrast, configuration 6 is interesting in Rayleigh channel because it does not only require the smallest energy but also lower SNR to obtain PER of $10^{-1}$ than configurations 2 and 3, and same SNR as configurations 4. Although it still requires a higher SNR than configuration 5 , it is worth noting that the coding rate of this configuration is highest than the one of configuration 5. In a general way, selecting a configuration with low data rate is better for AWGN channel in terms of minimum SNR required to obtain a target PER at the receiver. Meanwhile, configurations with higher data rate are good choices for deep fading channels, particularly in NLoS environment.

\section{CONCLUSIONS}

The LoRa standard considers different degrees of freedom to configure the transmission and includes the ADR mechanism to dynamically adapt the data-rates to the channel conditions. This algorithm is based on the received SNR to change the configuration. In this paper, we analyzed the trade-off between reliability and transmitter energy consumption according to channel parameters. In this context, we used simulation to

In addition, the transmitter energy consumption was studied for a Semtech device with three different values of transmit power. According to a target PER, we are able to connect the required SNR with the energy consumption for all configurations. One can then choose the best configurations according evaluate the reliability of LoRa modulation in a Rice fading channel (two parameters $K$ factor and node speed were tuned). The PER was considered as a QoS criterion and six different configurations were selected (spreading factor, bandwidth, code rate). Simulation results showed that a high SF enhances the performance in AWGN channel. However, it is also more sensitive to channel variations, which is illustrated by the higher loss of received signal power to maintain the same PER as in AWGN channel.

to a deployment. Ideally, the configuration may be chosen including not only SNR but also fading and velocity.

\section{REFERENCES}

[1] LoRa Alliance, "White paper: A technical overview of LoRa and LoRaWAN," The LoRa Alliance: San Ramon, Tech. Rep., 2015.

[2] K. E. Nolan, W. Guibene, and M. Y. Kelly, "An evaluation of low power wide area network technologies for the Internet of Things," in International Wireless Communications and Mobile Computing Conference (IWCMC), Sept. 2016, pp. 439-444.

[3] A. Augustin, J. Yi, T. Clausen, and W. M. Townsley, "A study of LoRa: long range and low power networks for the Internet of Things," Sensors, no. 2016: 1466

[4] F. Ait Aoudia, M. Gautier, M. Magno, M. Le Gentil, O. Berder, and L. Benini, "Long-short range communication network leveraging LoRa and wake-up receiver," Microprocessors and Microsystems, vol. 56, pp. $184-192,2018$

[5] J. Petjjrvi, K. Mikhaylov, M. Pettissalo, J. Janhunen, and J. Iinatti, "Performance of a low-power wide-area network based on LoRa technology: Doppler robustness, scalability, and coverage," International Journal of Distributed Sensor Networks, vol. 13, no. 3, 2017.

[6] F. Adelantado, X. Vilajosana, P. Tuset-Peiro, B. Martinez, J. MeliaSegui, and T. Watteyne, "Understanding the Limits of LoRaWAN," IEEE Communications Magazine, vol. 55, no. 9, pp. 34-40, 2017.

[7] F. Ait Aoudia, M. Magno, M. Gautier, O. Berder, and L. Benini, "A Low Latency and Energy Efficient Communication Architecture for Heterogeneous Long-Short Range Communication," in Euromicro Conference on Digital System Design (DSD), Limassol, Cyprus, Aug. 2016.

[8] O. Seller and N. Sornin, "Low power long range transmitter," Aug. 2014, US Patent App. 14/170,170. [Online]. Available: https://www.google.com/patents/US20140219329

[9] Semtech, "SX1272/73-860 MHz to $1020 \mathrm{MHz}$ low power long range transceiver," Tech. Rep. Datasheet, Mar. 2015. [Online]. Available: http://www.semtech.com/images/datasheet/sx1272.pdf

[10] A. Springer, W. Gugler, M. Huemer, L. Reindl, C. C. W. Ruppel, and R. Weigel, "Spread spectrum communications using chirp signals," in IEEE/AFCEA EUROCOMM 2000, Information Systems for Enhanced Public Safety and Security, 2000, pp. 166-170.

[11] B. Reynders and S. Pollin, "Chirp spread spectrum as a modulation technique for long range communication," in Symposium on Communications and Vehicular Technologies (SCVT), Nov. 2016, pp. 1-5.

[12] W. Jakes, Microwave mobile communications, ser. IEEE Press classic reissue. IEEE Press, 1974.

[13] T. Aulin, "A modified model for the fading signal at a mobile radio channel," IEEE Transactions on Vehicular Technology, vol. 28, no. 3, pp. 182-203, Aug. 1979. 